\title{
The clinical utility of transperineal template- guided saturation prostate biopsy for risk stratification after transrectal ultrasound-guided biopsy
}

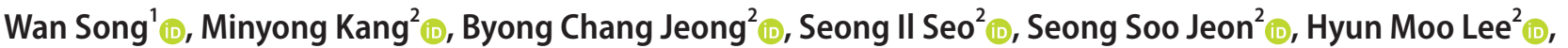 \\ Hwang Gyun Jeon ${ }^{2}$ (ID \\ 'Department of Urology, Ewha Womans University Medical Center, Ewha Womans University School of Medicine, Seoul, '2Department of Urology, Samsung Medical \\ Center, Sungkyunkwan University School of Medicine, Seoul, Korea
}

Purpose: To investigate the clinical utility of transperineal template-guided saturation prostate biopsy (TPB) for risk stratification after transrectal ultrasound (TRUS)-guided biopsy.

Materials and Methods: We retrospectively reviewed 155 patients who underwent TPB after previously negative results on TRUSguided biopsy $(n=58)$ or who were candidates for active surveillance $(n=97)$ fulfilling the PRIAS criteria between May 2017 and November 2018. The patients' clinicopathologic data were reviewed, and the detection of clinically significant cancer (CSC) and upgrading of Gleason grade were identified.

Results: The patients' median age and pre-TPB prostate-specific antigen (PSA) value were 65.0 years and $5.74 \mathrm{ng} / \mathrm{mL}$, respectively. A median of 36 biopsy cores was obtained in each patient, with a median TPB core density of $0.88 \mathrm{cores} / \mathrm{cm}^{3}$. Of the 58 males with a previous negative result on TRUS-guided biopsy, prostate cancer (PCa) was detected in 17 males (29.3\%), including 8 with CSC. Of the 97 patient candidates for active surveillance, upgrading of the Gleason grade was identified in 31 males (32.0\%), 20 with a Gleason grade of $7(3+4), 6$ with a Gleason grade of $7(4+3)$, and 5 with a Gleason grade of $8(4+4)$. The overall complication rate was $14.8 \%$ (23/155), and there were no Clavien-Dindo grade 3 to 5 complications.

Conclusions: TPB helps to stratify the risk of PCa that was previously missed or underdiagnosed by TRUS-guided biopsy. TPB might be used as a diagnostic tool to determine risk classification and to help counsel patients with regard to treatment decisions.

Keywords: Biopsy; Prostatic neoplasms; Risk; Watchful waiting

This is an Open Access article distributed under the terms of the Creative Commons Attribution Non-Commercial License (http://creativecommons.org/licenses/by-nc/4.0) which permits unrestricted non-commercial use, distribution, and reproduction in any medium, provided the original work is properly cited.

Received: 11 February, 2019 • Accepted: 8 July, 2019

Corresponding Author: Hwang Gyun Jeon (iD https://orcid.org/0000-0002-5613-8389

Department of Urology, Samsung Medical Center, Sungkyunkwan University School of Medicine, 81 Irwon-ro, Gangnam-gu, Seoul 06351, Korea

TEL: +82-2-2148-9599, FAX: +82-2-3410-6992, E-mail: hwanggyun.jeon@samsung.com 


\section{INTRODUCTION}

A standard 10- to 12-core transrectal ultrasound (TRUS)guided biopsy is the most commonly used diagnostic modality for the detection of prostate cancer $(\mathrm{PCa})[1,2]$. However, there is a chance of undetected PCa, and even high-grade $\mathrm{PCa}$, in some males with a previous negative result on TRUS-guided biopsy. In addition, although active surveillance (AS) has emerged as a treatment strategy in males with low-risk and very-low-risk PCa [3-5], TRUS-guided biopsy can miss high-grade or high-volume PCa and is not suitable for AS because of undersampling in the anterior prostate [6,7]. As a result, a sizeable minority of males initially classified as having low-risk or very-low-risk PCa ultimately receive definite treatment including radical prostatectomy (RP) or progress to metastatic disease and die from PCa $[5,8,9]$. Consequently, TRUS-guided biopsy alone is not sufficient, and a more reliable diagnostic modality that accurately categorizes risk stratification is needed.

Transperineal template-guided saturation prostate biopsy (TPB) is an alternative diagnostic modality for sampling prostate tissues. TPB has the advantage of being able to make a reliable approach to the entire prostate gland, including the anterior prostate and the transitional zone [10]. In addition, because TPB is usually conducted under general anesthesia, during which the prostate is not mobilized, a larger number of cores are obtained while confirming each biopsy position in three dimensions [6,11,12]. Therefore, the outcomes of TPB more precisely reflect subsequent pathologic results of RP, as opposed to those from TRUS-guided biopsy [13,14]. However, the clinical implications of TPB in patients with negative results on TRUS-guided biopsy or in those who were candidates for AS have not been fully investigated.

AS for localized PCa requires more precise risk stratification, and detection of missed $\mathrm{PCa}$ in patients with negative results on TRUS-guided biopsy is crucial to provide effective treatment options and to predict prognosis. Therefore, in this study, we investigated the clinical utility of TPB for risk stratification after TRUS-guided biopsy in patients with negative results on TRUS-guided biopsy or who were candidates for AS.

\section{MATERIALS AND METHODS}

\section{Study population}

This study was approved by the Institutional Review Board of Samsung Medical Center (approval number: 201805-192) and was performed in accordance with the prin- ciples of the Declaration of Helsinki. The requirement for informed consent was waived owing to the study design. We retrospectively reviewed a database of 155 consecutive male patients who underwent TPB between May 2017 and November 2018. The indications for TPB were as follows: 1) a persistent suspicion of $\mathrm{PCa}$ with rising prostate-specific antigen (PSA) despite a negative result on TRUS-guided biopsy $(n=58)$ or 2$)$ a confirmed or scheduled biopsy in male candidates for AS after TRUS-guided biopsy ( $n=97)$. The eligibility criteria for AS were biopsy Gleason grade $6(3+3)$, PSA $\leq 10 \mathrm{ng} / \mathrm{mL}$, clinical stage T1c/T2, PSA density (PSAD) $<0.2 \mathrm{ng} / \mathrm{mL} / \mathrm{cm}^{3}$, two or fewer positive cores $(\mathrm{n}=89)$ [8], and Gleason grade $\leq 7(3+4)(n=8)$. Multiparametric magnetic resonance imaging (mpMRI) was performed in 133 patients before TPB and was scored by using the Prostate Imaging Reporting and Data System version 2 (PI-RADSv2), except in one patient because of the poor quality of the images obtained.

\section{Data collection}

The clinical, laboratory, and pathologic data of the male patients, including age, PSA, prostate volume, PSAD, number of total and positive cores, and Gleason grade, were reviewed from the electronic medical records of our database. Gleason grade of TRUS-guided biopsy and TPB were examined by the same pathologist who specialized in genitourinary cancer. After TPB, the bilaterality of PCa was examined, and upgrading of Gleason grade was defined as an increase from Gleason grade 6 at TRUS-guided biopsy to Gleason grade 7 $(3+4)$ or greater after TPB. Clinically significant cancer (CSC) was defined as PCa with Gleason grade $7(3+4)$ or greater.

\section{MRI protocol and interpretation}

mpMRI was performed by using a $3.0 \mathrm{~T}$ MRI instrument (Intera Achieva TX; Philips Healthcare, Best, The Netherlands) with a six-channel, phase-array body coil. The protocols for mpMRI were based on the European Society of Urogenital Radiology guidelines [15] and included T1-weighted, T2-weighted, and diffusion-weighted imaging with $b$ values of 0,100 , and $1,000 \mathrm{~s} / \mathrm{mm}^{2}$, and dynamic contrast-enhanced imaging after intravenous injection of gadolinium diethylenetriamine pentaacetic acid (Gadovist; Schering, Berlin, Germany).

The MR images were loaded by using a picture archiving and communication system (Centricity; GE Healthcare, Barrington, $\mathrm{L}, \mathrm{USA}$ ) and were reviewed by a radiologist with more than 10 years of experience in prostate MRI interpretation who was blind to the clinical and pathologic information. The reviewer scored an index lesion with PI- 
RADSv2 by using a 5-point scale in each patient. PI-RADSv2 assessment categories were defined as follows: score 1, clinically significant cancer is highly unlikely to be present; score 2, clinically significant cancer is unlikely to be present; score 3 , the presence of clinically significant cancer is equivocal; score 4, clinically significant cancer is likely to be present; and score 5, clinically significant cancer is highly likely to be present [10].

\section{TPB technique}

Prior to TPB, a blood test for prothrombin time and activated partial thromboplastic time was examined to evaluate the coagulation profile. Patients were asked to stop oral anticoagulants for 1 week before the biopsy. All biopsies were performed on an inpatient procedure. Patients received Yal solution (docusate sodium $10 \mathrm{mg}$ ) and a bisacodyl suppository as a rectal preparation. For prophylactic treatment, oral cephalosporin and/or quinolone was given for 1 week from the day before the biopsy, and aminoglycoside (amikacin 500 $\mathrm{mg}$ ) was administered intramuscularly a few minutes before biopsy.

TPB was performed with the patients under general anesthesia to immobilize the prostate during the procedure. Patients were repositioned to an extended lithotomy position (Fig. 1A), and a Foley catheter was inserted. A digital rectal examination was performed. Then, TPB was performed using a biplanar TRUS 8848 brachytherapy probe (BK Medical, Herlev, Denmark) covered with a water-filled brachy- therapy balloon and Classic STEPPER ${ }^{\mathrm{TM}}$ device (CIVCO, Kalona, IA, USA) (Fig. 1B). Systemic biopsies were collected through the perineum using an 18-G, 20-cm long biopsy needle (ACECUT; TSK Laboratory, Tochigi, Japan) via a standard brachytherapy grid at 5-mm intervals, from the medial to the lateral plane in the anterior, mid, and posterior sectors (Fig. 1C), which were divided into right and left sides [16,17]. Depending on the distance from the prostate apex to the base, a single longitudinal biopsy was performed when the prostate length was less than $2 \mathrm{~cm}$, whereas double longitudinal biopsies were conducted when the length was more than $2 \mathrm{~cm}$ (Fig. 1D). As such, the number of biopsy cores obtained was directly dependent on the size of the prostate. The patients were discharged the morning after the procedure, after confirmation that they could pass urine.

\section{Statistical analysis}

Quantitative variables are presented as medians (range) or means \pm standard deviation, and qualitative variables are presented as absolute values (percentage). Descriptive statistics were obtained for demographic variables. All statistical analyses were performed by using IBM SPSS for Windows, version 23.0 (IBM Corp., Armonk, NY, USA).

\section{RESULTS}

The baseline demographics and characteristics of the 155 patients who underwent TPB are summarized in Table 1.
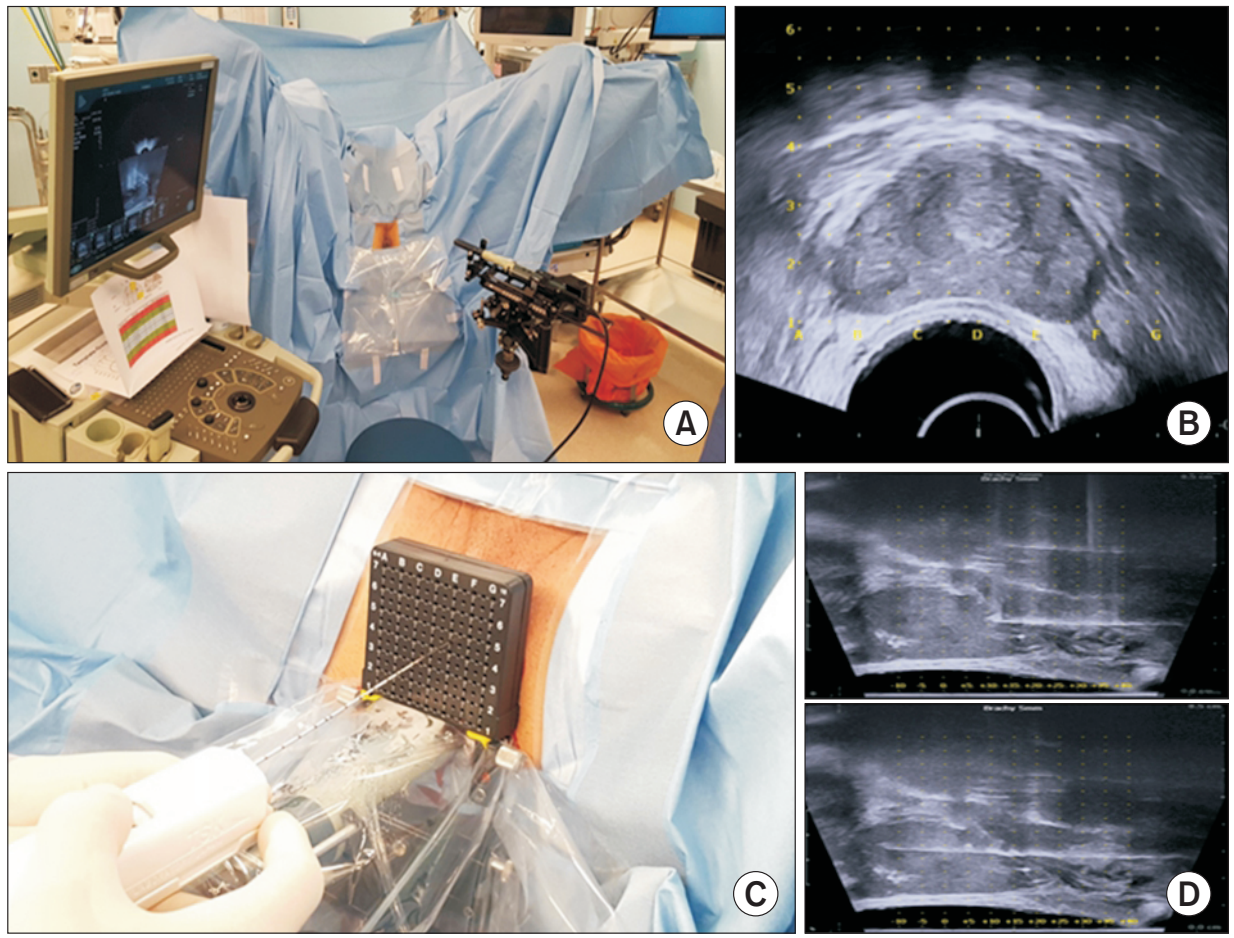

(C)

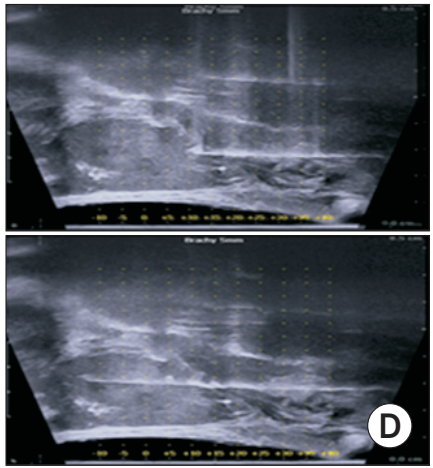

Fig. 1. Transperineal template-guided saturation prostate biopsy. (A) Patient positioning, (B) size measurement and setting, $(C)$ systemic biopsies via a standard brachytherapy grid, and (D) sagittal view of longitudinal biopsy. 
Table 1. Patient demographics and characteristics at baseline

\begin{tabular}{|c|c|c|c|}
\hline Variable & Total & $\begin{array}{l}\text { Previously negative } \\
\text { TRUS-guided biopsies }\end{array}$ & $\begin{array}{c}\text { Candidates for } \\
\text { active surveillance }\end{array}$ \\
\hline No. of patients, $\mathrm{n}(\%)$ & $155(100.0)$ & $58(37.4)$ & $97(62.6)$ \\
\hline \multicolumn{4}{|l|}{ Age at biopsy (y) } \\
\hline Median (range) & $65.0(47.0-79.0)$ & $64.0(51.0-77.0)$ & $67.0(47.0-79.0)$ \\
\hline Mean $\pm S D$ & $65.2 \pm 7.2$ & $63.9 \pm 6.5$ & $66.0 \pm 7.6$ \\
\hline \multicolumn{4}{|l|}{ PSA (ng/mL) } \\
\hline Median (range) & $5.74(0.56-42.76)$ & $6.47(1.05-42.76)$ & $5.55(0.56-23.47)$ \\
\hline Mean \pm SD & $7.63 \pm 6.05$ & $9.10 \pm 8.26$ & $6.75 \pm 4.01$ \\
\hline \multicolumn{4}{|l|}{ Prostate volume $\left(\mathrm{cm}^{3}\right)$} \\
\hline Median (range) & $41.0(12.3-233.0)$ & $44.6(17.5-233.0)$ & $37.9(12.3-107.4)$ \\
\hline Mean \pm SD & $45.7 \pm 24.9$ & $50.7 \pm 32.2$ & $42.7 \pm 19.0$ \\
\hline \multicolumn{4}{|l|}{ PSA density $\left(\mathrm{ng} / \mathrm{mL} / \mathrm{cm}^{3}\right)$} \\
\hline Median (range) & $0.15(0.04-0.96)$ & $0.15(0.04-0.96)$ & $0.15(0.04-0.71)$ \\
\hline Mean \pm SD & $0.19 \pm 0.14$ & $0.18 \pm 0.14$ & $0.19 \pm 0.13$ \\
\hline \multicolumn{4}{|c|}{ Pathologic results in TRUS-guided biopsy ${ }^{\mathrm{a}}, \mathrm{n}(\%)$} \\
\hline HGPIN & & $7(12.1)$ & \\
\hline ASAP & & $5(8.6)$ & \\
\hline Benign prostatic tissue & & $46(79.3)$ & \\
\hline \multicolumn{4}{|c|}{ No. of total cores in TRUS-guided biopsy ${ }^{b}$} \\
\hline Median (range) & & & $12.0(4.0-15.0)$ \\
\hline Mean \pm SD & & & $12.0 \pm 1.4$ \\
\hline \multicolumn{4}{|c|}{ No. of positive cores in TRUS-guided biopsy ${ }^{b}$} \\
\hline Median (range) & & & $1.0(1.0-8.0)$ \\
\hline Mean $\pm S D$ & & & $1.5 \pm 1.1$ \\
\hline \multicolumn{4}{|c|}{ Gleason grade in TRUS-guided biopsy ${ }^{\mathrm{b}}, \mathrm{n}(\%)$} \\
\hline 6 & & & $89(91.8)$ \\
\hline $7(3+4)$ & & & $8(8.2)$ \\
\hline
\end{tabular}

TRUS, transrectal ultrasonography; SD, standard deviation; PSA, prostate-specific antigen; HGPIN, high-grade prostatic intraepithelial neoplasia; ASAP, atypical small acinar proliferation.

${ }^{a} \mathrm{n}=58,{ }^{\mathrm{b}} \mathrm{n}=97$.

The patients' median (range) age at TPB was 65.0 (47.0-79.0) years. Median PSA and prostate volumes were 5.74 (0.5642.76) $\mathrm{ng} / \mathrm{mL}$ and $41.0(12.3-233.0) \mathrm{cm}^{3}$, respectively. Of the 97 candidates for AS, Gleason grade in TRUS-guided biopsy was $6(3+3)$ in 89 patients $(91.8 \%)$ and $7(3+4)$ in 8 patients $(8.2 \%)$.

The results of TPB are presented in Table 2 . The median (range) duration of TPB was 25 (15.0-40.0) minutes. The median (range) number of TPB cores was 36.0 (13.0-40.0), and PCa was identified in 85 patients (54.8\%) overall. Of the 58 males with a previous negative result on TRUS-guided biopsy, PCa was detected in 17 (29.3\%), including 8 with CSC. Of the 97 candidates for AS, PCa was detected in 68 (70.1\%); overall, the Gleason grade of 31 patients (32.0\%) was upgraded after TPB, including 20 with Gleason grade $7(3+4), 6$ with Gleason grade $7(4+3)$, and 5 with Gleason grade $8(4+4)$. In addition, a total of 40 patients (41.2\%) were diagnosed with bilateral PCa.
The total complication rate after TPB was 16.8\% (26/155), including acute urinary retention $(12.3 \%, 19 / 155)$ that required temporary Foley catheter indwelling, post-voiding residual urine $(0.6 \%, 1 / 155)$ that required clean intermittent catheterization, gross hematuria $(3.2 \%, 5 / 155)$ with overnight bladder irrigation, and hematospermia $(0.6 \%, 1 / 155)$. There were no Clavien-Dindo grade 3 to 5 complications, such as transfusion, acute prostatitis, or readmission.

Pathologic correlation between TPB and RP specimens is summarized in Table 3. After TPB, a total of 30 patients underwent RP, and upgrading of Gleason grade was identified in 5 patients (16.7\%). However, 20 patients (66.7\%) showed the same Gleason grade of TPB after RP.

The outcomes of TPB stratified by PI-RADSv2 score in mpMRI are depicted in Fig. 2. Of the 39 patients with a previously negative result on TRUS-guided biopsy, a PIRADSv2 score of 1 to 3 was given in 35 (89.7\%), and PCa was detected in 7 (20.0\%), including 3 with a Gleason grade of 7 
Table 2. Results of transperineal template-guided saturation prostate biopsies and PI-RADSv2 scores

\begin{tabular}{|c|c|c|c|}
\hline Variable & Total & $\begin{array}{l}\text { Previously negative } \\
\text { TRUS-guided biopsies }\end{array}$ & $\begin{array}{c}\text { Candidates for } \\
\text { active surveillance }\end{array}$ \\
\hline No. of patients, $\mathrm{n}(\%)$ & $155(100.0)$ & $58(37.4)$ & $97(62.6)$ \\
\hline No. of patients with positive TPB, $n$ (\%) & $85(54.8)$ & $17(29.3)$ & $68(70.1)$ \\
\hline \multicolumn{4}{|l|}{ No. of total cores } \\
\hline Median (range) & $36.0(13.0-40.0)$ & $36.0(24.0-40.0)$ & $36.0(13.0-40.0)$ \\
\hline Mean \pm SD & $32.8 \pm 5.8$ & $32.7 \pm 5.7$ & $32.9 \pm 5.8$ \\
\hline \multicolumn{4}{|l|}{ No. of positive cores ${ }^{a}$} \\
\hline Median (range) & $4.0(1.0-26.0)$ & $3.0(1.0-15.0)$ & $4.0(1.0-26.0)$ \\
\hline Mean $\pm S D$ & $5.0 \pm 4.4$ & $3.7 \pm 3.7$ & $5.3 \pm 4.6$ \\
\hline \multicolumn{4}{|l|}{ Detection of $\operatorname{CSC}^{\mathrm{a}}, \mathrm{n}(\%)$} \\
\hline Yes & $39(45.9)$ & $8(47.1)$ & $31(45.6)$ \\
\hline No & $46(54.1)$ & $9(52.9)$ & $37(54.4)$ \\
\hline \multicolumn{4}{|l|}{ Bilateral detection of prostate cancer ${ }^{\mathrm{a}}, \mathrm{n}(\%)$} \\
\hline Yes & $48(56.5)$ & $8(47.1)$ & $40(58.8)$ \\
\hline No & $37(43.5)$ & $9(52.9)$ & $28(41.2)$ \\
\hline \multicolumn{4}{|l|}{ Gleason grade in TPB, $\mathrm{n}(\%)$} \\
\hline Benign & $70(45.1)$ & $41(70.7)$ & $29(29.9)$ \\
\hline 6 & $46(29.7)$ & $9(15.5)$ & $37(38.1)$ \\
\hline $7(3+4)$ & $24(15.5)$ & $4(6.9)$ & $20(20.6)$ \\
\hline $7(4+3)$ & $7(4.5)$ & $1(1.7)$ & $6(6.2)$ \\
\hline 8 & $8(5.2)$ & $3(5.2)$ & $5(5.2)$ \\
\hline \multicolumn{4}{|l|}{ PI-RADSv2 score ${ }^{\mathrm{b}}, \mathrm{n}(\%)$} \\
\hline $1-3$ & $77(58.3)$ & $35(89.7)$ & $42(45.2)$ \\
\hline $4-5$ & $55(41.7)$ & $4(10.3)$ & $51(54.8)$ \\
\hline
\end{tabular}

PI-RADSv2, Prostate Imaging Reporting and Data System version 2; TRUS, transrectal ultrasonography; TPB, transrectal template-guided saturation prostate biopsy; SD, standard deviation; CSC, clinically significant prostate cancer.

${ }^{a} \mathrm{n}=85,{ }^{\mathrm{b}} \mathrm{n}=132$.

Table 3. Comparison of Gleason grade after transperineal template-guided saturation prostate biopsy and pathologic results after radical prostatectomy

\begin{tabular}{|c|c|c|c|c|c|}
\hline & \multicolumn{5}{|c|}{ Gleason grade after RP } \\
\hline & $6(3+3)$ & $7(3+4)$ & $7(4+3)$ & $\geq 8$ & Total \\
\hline \multicolumn{6}{|c|}{ Gleason grade after TPB } \\
\hline $6(3+3)$ & $5(16.7)$ & $4(13.3)$ & 0 & 0 & $9(30.0)$ \\
\hline $7(3+4)$ & $1(3.3)$ & $15(50.0)$ & 0 & $1(3.3)$ & $17(56.7)$ \\
\hline $7(4+3)$ & 0 & $1(3.3)$ & 0 & 0 & $1(3.3)$ \\
\hline$\geq 8$ & 0 & $1(3.3)$ & $2(6.7)$ & 0 & $3(10.0)$ \\
\hline Total & $6(20.0)$ & $21(70.0)$ & $2(6.7)$ & $1(3.3)$ & $30(100.0)$ \\
\hline
\end{tabular}

Values are presented as number (\%).

$\mathrm{RP}$, radical prostatectomy; TPB, transrectal template-guided saturation prostate biopsy.

$(3+4)$. Of the remaining 4 patients with a PI-RADSv2 score of 4 to 5 , PCa was found in $3(75.0 \%)$, and one had Gleason grade $8(4+4)$. Of the 85 candidates for AS with Gleason grade $6(3+3)$, a PI-RADSv2 score of 1 to 3 was identified in 38 (44.7\%), in whom PCa was detected in 20 (52.6\%), including 11 (28.9\%) who were upgraded to Gleason grade $7(3+4)$ or greater. When the PI-RADSv2 score was 4 to 5 , 38 patients (80.9\%) were diagnosed with PCa, of whom 15 (31.9\%) were upgraded to Gleason grade $7(3+4)$ or greater after TPB.

The treatment strategy in patients who were candidates for AS according to the results of TPB is shown in Fig. 3. Of the total 97 patients, 29 (29.9\%) with benign pathology at TPB remained on AS. Of the 37 (38.1\%) with Gleason grade 6 (3+3), AS or definite treatment including RP could be considered when cancer was identified bilaterally $(n=17,17.5 \%)$, whereas focal therapy was considered if cancer was con- 

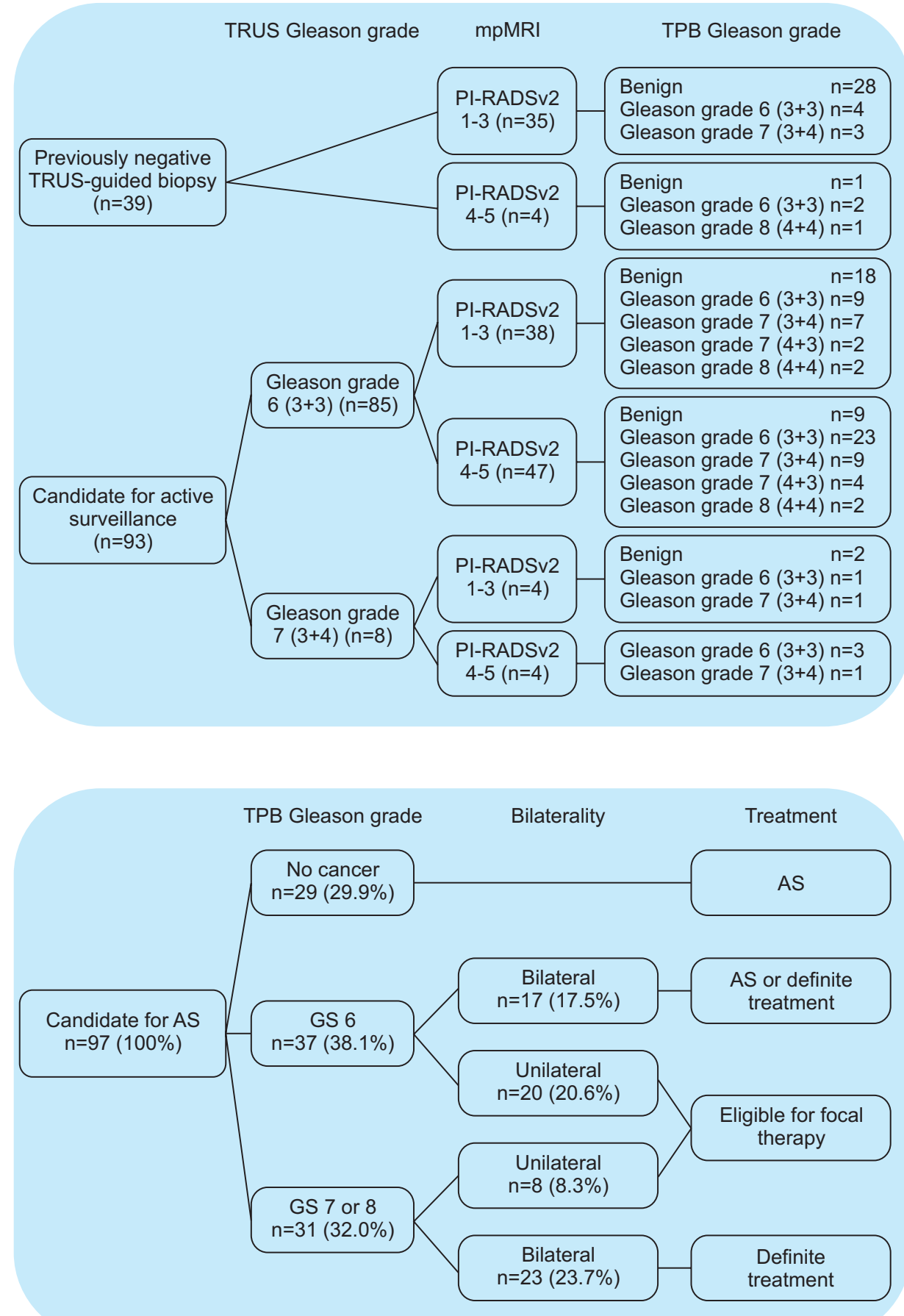

Fig. 2. Association between Prostate Imaging Reporting and Data System version 2 (PI-RADSv2) score and results of transperineal template-guided saturation prostate biopsy (TPB) in males with a previous negative result on transrectal ultrasound (TRUS)-guided biopsy or who were candidates for active surveillance. mpMRI, multiparametric magnetic resonance imaging.

Fig. 3. Treatment strategy for patients with active surveillance (AS) according to transperineal template-guided saturation prostate biopsy (TPB). GS, Gleason score. firmed unilaterally ( $\mathrm{n}=20,20.6 \%)$. Of the remaining 31 males (32.0\%) with Gleason grade 7 or 8, definite treatment was required. However, when an indication for focal therapy was expanded to males with a unilaterally identified Gleason grade of 7 (3+4), 8 additional males (8.3\%) were included, and 28 (28.9\%) were eligible for focal therapy overall.

\section{DISCUSSION}

In this study, of the 58 patients with negative results on
TRUS-guided biopsy, PCa was identified in 17 (29.3\%), including 8 with Gleason grade $7(3+4)$ or greater. In addition, of the 97 candidates for AS, an upgraded Gleason grade was identified in 31 (32.0\%). Furthermore, in 28 patients (28.9\%) with unilaterally identified PCa with Gleason grade $6(3+3)$ or $7(3+4)$, focal therapy could be considered. These results suggest that TPB can classify the risk of PCa previously missed or underdiagnosed by TRUS-guided biopsy. Therefore, TPB can be used as a diagnostic tool to determine risk stratification of $\mathrm{PCa}$ and can be used to help counsel pa- 
tients with regard to treatment strategy. To the best of our knowledge, this is the first study to analyze the clinical utility of TPB for risk stratification after TRUS-guided biopsy in Korean males.

The widespread use of PSA screening for PCa has led to the detection of low-risk and very-low-risk $\mathrm{PCa}$, and these patients are eligible for various treatment options other than RP, including AS or focal therapy [18]. However, approximately one-third of males on AS ultimately have experienced delayed treatment within the first few years [6], and misclassification at diagnosis was the most likely cause of treatment failure rather than disease progression [11,19]. Therefore, accurate diagnosis of PCa is essential for optimal patient selection, and a more dedicated biopsy strategy to compensate for the insufficiency of existing diagnostic tools is required.

In our study, PCa was identified in 17 patients (29.3\%), including 8 with CSC, after TPB in 58 patients with a previous negative result on TRUS-guided biopsy. There is no consensus for repeat biopsy strategies after an initial negative result. In a study by Nafie et al. [20], 42 patients with previous negative results on TRUS-guided biopsy underwent simultaneous 12-core TRUS and 36-core perineal biopsy. In that study, TPB showed a significantly higher PCa detection rate than did TRUS-guided biopsy ( $45 \%$ vs. $15 \%$, $\mathrm{p}=0.01$ ). However, that meta-analysis study, which included 12 studies of TRUS-guided biopsy and 14 studies of TPB as a repeat biopsy strategy, reported that cancer detection rates were $30.0 \%$ and $36.8 \%$ for TRUS-guided biopsy and TPB, respectively [21].

In addition, several studies that have analyzed the role of TPB for risk stratification after TRUS-guided biopsy in patients for AS are summarized in Table 4 [6,11,12,16,22-25].
Of the eight recently published studies, a commonly reported significant rate for tumor upgrading after TPB ranged from $29.2 \%$ to $41.7 \%$ [6,11,12,16,22-25]. In our study, $32.0 \%$ of patients (31/97) were upgraded after TPB, and the median TPB core density (a ratio of the number of biopsy cores to prostate volume) was 0.88 cores $/ \mathrm{cm}^{3}$, which suggests that the prostate was comprehensively sampled. Although the results of our study agreed with those of previous studies, our rate of upgrading was on the lower end of the spectrum because the criteria for eligibility for AS were more rigorous than in other studies. In a study by Voss et al. [11], a total of 208 patients (Gleason score $\leq 3+4=7$, PSA $\leq 15 \mathrm{ng} / \mathrm{mL}$, T1-T2, and $\leq 50 \%$ positive cores) enrolled in AS underwent TPB, and $39.9 \%$ (83/208) were upgraded. However, when restricted to the most strict Epstein criteria [26], the rate of upgrading decreased to $29.0 \%$, which is similar to our results. Taking all these results into account, TPB could be considered in patients with suspected $\mathrm{PCa}$ due to persistent elevation of PSA after a negative result on TRUS-guided biopsy or in candidates for AS for risk confirmation after TRUS-guided biopsy.

Recently, mpMRI scored with PI-RADSv2 has emerged as a promising modality in the diagnosis of $\mathrm{PCa}$, and PIRADSv2 scores of 4 to 5 were found to increase the predictive accuracy of upgrading [27]. In our study, of 55 patients with PI-RADSv2 scores of 4 to 5, PCa was confirmed in 45 (81.8\%), of which 17 (37.8\%) were upgraded to Gleason grade $7(3+4)$ or greater at TPB. Of 45 patients with $\mathrm{PCa}$, the concordance rate of location of the PCa lesion was $84.4 \%$ (38/45). However, there is a chance of missing invisible or unclear significant PCa in mpMRI. In fact, of the 77 males with a PI-RADSv2 score of 1 to 3, a total of 15 (19.5\%) were identified as harboring PCa with Gleason grade $7(3+4)$ or greater.

Table 4. Comparison of results from studies evaluating the role of transperineal template-guided saturation prostate biopsies in active surveillance

\begin{tabular}{|c|c|c|c|c|c|c|c|c|}
\hline Study & Location & $\begin{array}{c}\text { No. of } \\
\text { patients }\end{array}$ & $\begin{array}{l}\text { Median } \\
\text { age }(y)\end{array}$ & $\begin{array}{c}\text { Median PSA } \\
(\mathrm{ng} / \mathrm{mL})\end{array}$ & $\begin{array}{l}\text { Median prostate } \\
\text { volume }\left(\mathrm{cm}^{3}\right)\end{array}$ & $\begin{array}{l}\text { Median number } \\
\text { of cores }\end{array}$ & $\begin{array}{l}\text { Detection of } \\
\text { any cancer }\end{array}$ & Upgrading \\
\hline Voss et al. [11] & UK & 208 & 64.0 & 5.30 & 43.4 & 50.0 & $185(88.9)$ & $83(39.9)$ \\
\hline Hansen et al. [16] & UK & 132 & 66.0 & 7.30 & 50.0 & 26.0 & $110(83.3)$ & $55(41.7)$ \\
\hline \multirow[t]{2}{*}{ Pham et al. [23] } & USA & 46 & 66.0 & 4.90 & 44.0 & 24.0 & $35(76.1)$ & $16(34.8)$ \\
\hline & USA & 89 & 64.0 & 5.30 & 51.0 & 62.0 & 70 (78.7) & $26(29.2)$ \\
\hline Vyas et al. [25] & UK & 307 & 64.0 & 6.60 & 45.0 & 29.0 & $245(79.8)$ & $90(29.3)$ \\
\hline Radtke et al. [24] & Germany & 294 & 64.0 & 7.30 & 47.0 & 26.0 & $150(51.0)$ & $86(29.3)$ \\
\hline Merrick et al. [6] & USA & 131 & 65.7 & 4.80 & 46.0 & 50.8 & $104(79.4)$ & $52(39.7)$ \\
\hline Ayres et al. [22] & UK & 101 & 68.0 & 6.40 & 48.9 & 47.0 & $86(85.1)$ & $30(29.7)$ \\
\hline Taira et al. [12] & UK & 64 & 67.7 & 4.70 & 47.7 & 39.0 & $56(87.5)$ & $25(39.1)$ \\
\hline Present study & Korea & 97 & 67.0 & 5.55 & 37.9 & 36.0 & $68(70.1)$ & $31(32.0)$ \\
\hline
\end{tabular}

Values are presented as number only or number (\%). PSA, prostate-specific antigen. 
Therefore, when interpreting the PI-RADSv2 score, it is necessary to consider other clinicopathologic parameters, such as PSAD, number of positive cores, and maximum percentage of cancer per core [27].

Urinary retention, which is main complication, was identified in 19 patients (123\%) in our study. A recent systemic review reported that the occurrence of acute urinary retention was slightly higher after TPB, ranging from $1.7 \%$ to $11.1 \%$, than after TRUS-guided biopsy $(0.4 \%-6.0 \%)$ or MRIguided target biopsy ( $0 \%-1.0 \%$ ) [28]. However, urinary retention is usually transient and improves in most patients with the temporary placement of a Foley catheter.

Despite the potential clinical implications of our study, it had several limitations. First, our study included a relatively small number of patients, and a large, multi-institutional, and confirmatory study is warranted. However, our study was the first to evaluate the clinical utility of TPB in Korean males and provides a basis for further research. Second, we did not perform correlation analysis between the PIRADSv2 score and TPB results for index lesions. Furthermore, we did not have final pathologic results for patients who underwent definite treatment. However, a previous study reported that TPB accurately detected true PCa after RP [14]. Finally, because we did not have data on long-term follow-up, we could not confirm whether the detection of more aggressive cancer resulted in an improvement of prognosis, such as cancer-specific survival.

\section{CONCLUSIONS}

After TPB, 29.3\% of males with negative results on TRUS-guided biopsy were diagnosed with $\mathrm{PCa}$, and $32.0 \%$ of males who were candidates for AS showed upgrading of Gleason grade. Therefore, TPB stratifies the risk of PCa that was previously missed or underdiagnosed by TRUS-guided biopsy. TPB could be used as a diagnostic tool to determine risk classification and to help counsel patients with regard to treatment decisions.

\section{CONFLICTS OF INTEREST}

The authors have nothing to disclose.

\section{REFERENCES}

1. Loeb S, Vellekoop A, Ahmed HU, Catto J, Emberton M, Nam $\mathrm{R}$, et al. Systematic review of complications of prostate biopsy. Eur Urol 2013;64:876-92.

2. Song W, Choo SH, Sung HH, Han DH, Jeong BC, Seo SI, et al. Incidence and management of extended-spectrum betalactamase and quinolone-resistant Escherichia coli infections after prostate biopsy. Urology 2014;84:1001-7.

3. Mottet N, Bellmunt J, Bolla M, Briers E, Cumberbatch MG, De Santis M, et al. EAU-ESTRO-SIOG Guidelines on prostate cancer. Part 1: screening, diagnosis, and local treatment with curative intent. Eur Urol 2017;71:618-29.

4. Hayes JH, Ollendorf DA, Pearson SD, Barry MJ, Kantoff PW, Stewart ST, et al. Active surveillance compared with initial treatment for men with low-risk prostate cancer: a decision analysis. JAMA 2010;304:2373-80.

5. Klotz L, Zhang L, Lam A, Nam R, Mamedov A, Loblaw A. Clinical results of long-term follow-up of a large, active surveillance cohort with localized prostate cancer. J Clin Oncol 2010;28:126-31.

6. Merrick GS, Delatore A, Butler WM, Bennett A, Fiano R, Anderson $\mathrm{R}$, et al. Transperineal template-guided mapping biopsy identifies pathologic differences between very-low-risk and low-risk prostate cancer: implications for active surveillance. Am J Clin Oncol 2017;40:53-9.

7. Duffield AS, Lee TK, Miyamoto H, Carter HB, Epstein JI. Radical prostatectomy findings in patients in whom active surveillance of prostate cancer fails. J Urol 2009;182:2274-8.

8. Bokhorst LP, Valdagni R, Rannikko A, Kakehi Y, Pickles T, Bangma $\mathrm{CH}$, et al. A decade of active surveillance in the PRIAS study: an update and evaluation of the criteria used to recommend a switch to active treatment. Eur Urol 2016;70:954-60.

9. Krakowsky Y, Loblaw A, Klotz L. Prostate cancer death of men treated with initial active surveillance: clinical and biochemical characteristics. J Urol 2010;184:131-5.

10. Park SY, Jung DC, Oh YT, Cho NH, Choi YD, Rha KH, et al. Prostate cancer: PI-RADS version 2 helps preoperatively predict clinically significant cancers. Radiology 2016;280:108-16.

11. Voss J, Pal R, Ahmed S, Hannah M, Jaulim A, Walton T. Utility of early transperineal template-guided prostate biopsy for risk stratification in men undergoing active surveillance for prostate cancer. BJU Int 2018;121:863-70.

12. Taira AV, Merrick GS, Bennett A, Andreini H, Taubenslag W, Galbreath RW, et al. Transperineal template-guided mapping biopsy as a staging procedure to select patients best suited for active surveillance. Am J Clin Oncol 2013;36:116-20.

13. Thompson JE, Hayen A, Landau A, Haynes AM, Kalapara A, Ischia J, et al. Medium-term oncological outcomes for extended vs saturation biopsy and transrectal vs transperineal biopsy in active surveillance for prostate cancer. BJU Int 2015;115:88491.

14. Crawford ED, Rove KO, Barqawi AB, Maroni PD, Werahera $\mathrm{PN}$, Baer CA, et al. Clinical-pathologic correlation between transperineal mapping biopsies of the prostate and three- 
dimensional reconstruction of prostatectomy specimens. Prostate 2013;73:778-87.

15. Barentsz JO, Richenberg J, Clements R, Choyke P, Verma S, Villeirs G, et al. ESUR prostate MR guidelines 2012. Eur Radiol 2012;22:746-57.

16. Hansen N, Patruno G, Wadhwa K, Gaziev G, Miano R, Barrett $\mathrm{T}$, et al. Magnetic resonance and ultrasound image fusion supported transperineal prostate biopsy using the Ginsburg protocol: technique, learning points, and biopsy results. Eur Urol 2016;70:332-40.

17. Crawford ED, Wilson SS, Torkko KC, Hirano D, Stewart JS, Brammell C, et al. Clinical staging of prostate cancer: a computer-simulated study of transperineal prostate biopsy. BJU Int 2005;96:999-1004.

18. Carroll PR, Parsons JK, Andriole G, Bahnson RR, Castle EP, Catalona WJ, et al. NCCN Guidelines insights: Prostate Cancer Early Detection, version 2.2016. J Natl Compr Canc Netw 2016;14:509-19.

19. Klotz L, Vesprini D, Sethukavalan P, Jethava V, Zhang L, Jain S, et al. Long-term follow-up of a large active surveillance cohort of patients with prostate cancer. J Clin Oncol 2015;33:272-7.

20. Nafie S, Wanis M, Khan M. The efficacy of transrectal ultrasound guided biopsy versus transperineal template biopsy of the prostate in diagnosing prostate cancer in men with previous negative transrectal ultrasound guided biopsy. Urol J 2017; 14:3008-12.

21. Nelson AW, Harvey RC, Parker RA, Kastner C, Doble A, Gnanapragasam VJ. Repeat prostate biopsy strategies after initial negative biopsy: meta-regression comparing cancer de- tection of transperineal, transrectal saturation and MRI guided biopsy. PLoS One 2013;8:e57480.

22. Ayres BE, Montgomery BS, Barber NJ, Pereira N, Langley SE, Denham $\mathrm{P}$, et al. The role of transperineal template prostate biopsies in restaging men with prostate cancer managed by active surveillance. BJU Int 2012;109:1170-6.

23. Pham KN, Porter CR, Odem-Davis K, Wolff EM, Jeldres C, Wei JT, et al. Transperineal template guided prostate biopsy selects candidates for active surveillance--how many cores are enough? J Urol 2015;194:674-9.

24. Radtke JP, Kuru TH, Boxler S, Alt CD, Popeneciu IV, Huettenbrink $\mathrm{C}$, et al. Comparative analysis of transperineal template saturation prostate biopsy versus magnetic resonance imaging targeted biopsy with magnetic resonance imaging-ultrasound fusion guidance. J Urol 2015;193:87-94.

25. Vyas L, Acher P, Kinsella J, Challacombe B, Chang RT, Sturch P, et al. Indications, results and safety profile of transperineal sector biopsies (TPSB) of the prostate: a single centre experience of 634 cases. BJU Int 2014;114:32-7.

26. Epstein JI, Walsh PC, Carmichael M, Brendler CB. Pathologic and clinical findings to predict tumor extent of nonpalpable (stage T1c) prostate cancer. JAMA 1994;271:368-74.

27. Song W, Bang SH, Jeon HG, Jeong BC, Seo SI, Jeon SS, et al. Role of PI-RADS version 2 for prediction of upgrading in biopsy-proven prostate cancer with Gleason score 6. Clin Genitourin Cancer 2018;16:281-7.

28. Borghesi M, Ahmed H, Nam R, Schaeffer E, Schiavina R, Taneja $\mathrm{S}$, et al. Complications after systematic, random, and image-guided prostate biopsy. Eur Urol 2017;71:353-65. 